\title{
Les peptides dérivés du proglucagon : maturations post-traductionnelles et leurs conséquences biologiques
}

Le proglucagon est un polypeptide de 160 acides aminés, synthétisé dans le pancréas endocrine, les muqueuses gastrique et intestinale, et le système nerveux central. Il s'agit d'un précurseur transformé par maturation protéolytique en différentes hormones actives dont la nature diffère selon les tissus. Dans le pancréas et l'estomac, le glucagon, actif sur la glycorégulation, est l'espèce majoritaire alors que dans la muqueuse intestinale et le système nerveux central sont libérées la glicentine et l'oxyntomoduline dont l'un des rôles pourrait être, en périphérie, de contrôler négativement la sécrétion acide par les glandes oxyntiques de l'estomac. Outre la maturation protéolytique dans les tissus producteurs, les hormones dérivées du proglucagon subissent des clivages spécifiques au niveau des tissus cibles et sont transformées ainsi en molécules actives dont les effets ou la spécificité peuvent être différents de ceux des hormones mères : le glucagon (19-29) module la concentration intracellulaire de $\mathrm{Ca}^{2+}$, alors que l'oxyntomoduline (19-37) serait le vrai produit actif sur la sécrétion acide, interagissant avec des récepteurs différents de ceux de l'hormone intacte.

\section{Dominique Bataille}

\section{ADRESSE ET TIRÉS À PART}

D. Bataille : directeur de recherches à l'Inserm. Centre Cnrs-Inserm de pharmacologieendocrinologie, rue de la Cardonille, 34094 es peptides de la famille du glucagon offrent un vaste champ à l'investigateur, les régulations biologiques dans lesquelles ces peptides sont impliqués allant du contrôle de la glycémie (avec son pendant pathologique, le diabète) à la régulation de la sécrétion acide gastrique (avec son pendant pathologique, l'ulcère). Le peptide chef de file, le glucagon, caractérisé moléculairement en 1957 [1] à partir d'une sous-fraction de la purification de l'insuline, pré- sente un vaste éventail de sites d'action. Ceux qui paraissent les plus physiologiques se situent dans le domaine de la régulation du métabolisme (foie, tissu adipeux, cellule $B$ pancréatique...), d'autres sont considérés (peut-être à tort) comme plus pharmacologiques (estomac, cœur et vaisseaux, rein...). Avant le clonage du gène codant pour ce peptide (figure 1), trois molécules contenant les 29 acides aminés (AA) du glucagon avaient été isolées et caractérisées : le glucagon lui-même (29 AA) 


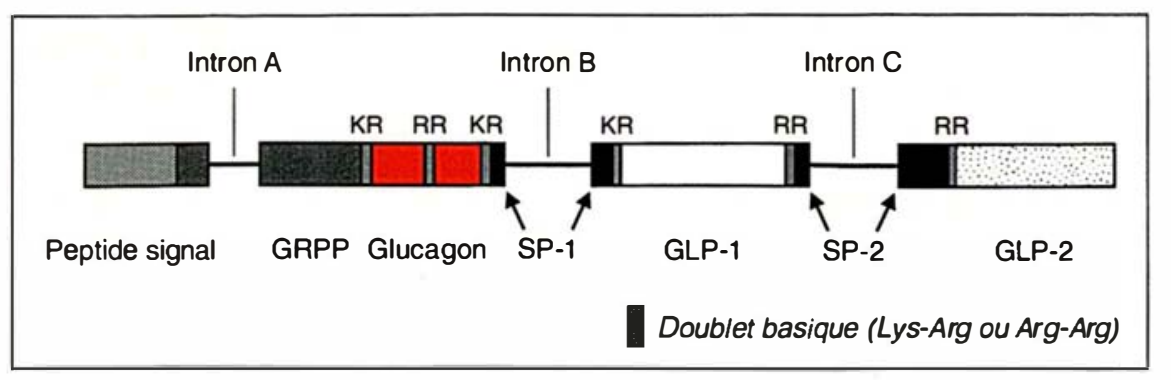

Figure 1. Structure de I'ADN génomique du préproglucagon humain. D'après Bell $[5,6]$. GRPP : glicentin-related pancreatic peptide ; SP-1 et -2: spacer peptides-1 et -2. GLP-1 et -2: glucagon-related peptides-1 et -2 . Les acides aminés basiques sont indiqués en code à une lettre : $K$ : lysine (Lys) ; $R$ : arginine (Arg).

[1], l'oxyntomoduline [2] (OXM, $37 \mathrm{AA})$, contenant un octapeptide Cterminal supplémentaire, et la glicentine [3] (69 AA), comportant les 37 AA de l'OXM plus un peptide $\mathrm{N}$-terminal de $32 \mathrm{AA}$ dont les 30 premiers AA correspondent au glicentin-related pancreatic peptide [4] (GRPP). La structure du précurseur après élimination du peptide signal de 20 AA (proglucagon) a révélé la présence de plusieurs domaines (figure 2), séparés par des doublets basiques (Lys-Arg, Arg-Arg ou ArgLys). Il convient de noter que, malgré quelques mutations, en particulier au niveau du GRPP qui ne semble pas remplir de rôle biologique spécifique, cette structure est très conservée chez les mammifères $[5,6]$. Outre le domaine contenant le glucagon (les 69 premiers AA du proglucagon correspondant à la structure de la glicentine), deux autres domaines ont été découverts dans cette structure, correspondant à deux peptides ressemblant au glucagon et nommés pour cette raison glucagon-like peptides I et $I I$ (GLP-I et GLP-II). Chez les vertébrés primitifs comme les poissons, un seul GLP existe [7]. Cette structure dérive, de toute évidence, de duplications du gène de départ suivies de mutations.

\section{Maturation post-traductionnelle dans les tissus producteurs}

Il nous a été possible d'analyser finement la maturation du proglucagon $\mathrm{m} / \mathrm{s} n^{\circ} 9$, vol. 7 , novembre 91 mammifères. culièrement intéressants pour étudier des processus de maturation, car l'épitope ciblé est masqué dans le précurseur et démasqué lors de la maturation recherchée. La spécificité du dosage est très grande car la présence d'un AA supplémentaire ou l'absence d'un AA en bout de chaîne fait chuter la reconnaissance par l'anticorps d'un facteur 100 à 1000 . Utilisant ces outils (figure 3, p. 902) couplés à la séparation des peptides contenus dans les extraits par chromatographie liquide à haute performance (CLHP), nous avons pu montrer que, chez le rat, deux types de maturation post-traductionnelle existent dans les tissus producteurs. Le premier type, trouvé dans le pancréas endocrine (en fait dans les cellules $\alpha$ produisant et sécrétant le glucagon) (figure 4, p. 903) et dans la muqueuse gastrique (qui, chez le rat, contient des cellules de type $\alpha$ ), correspond à la production du côté $\mathrm{N}$-terminal du peptide de $37 \mathrm{AA}$, l'oxyntomoduline, aux côtés du glucagon, peptide dominant dans ces tissus. Cela a été une surprise puisque ces données, non ambiguës, allaient à l'encontre de résultats $[3,10]$ selon lesquels le peptide de $69 \mathrm{AA}$ (glicentine) était le précurseur du glucagon. En fait, l'épitope de la glicentine reconnu par l'anticorps utilisé était contenu dans la partie $\mathrm{N}$-terminale de la molécule (GRPP), qui est clivée lors de la maturation du proglucagon, sans passer par un stade de glicentine. C'est ce GRPP qui a probablement été pris pour la glicentine dans des tra-

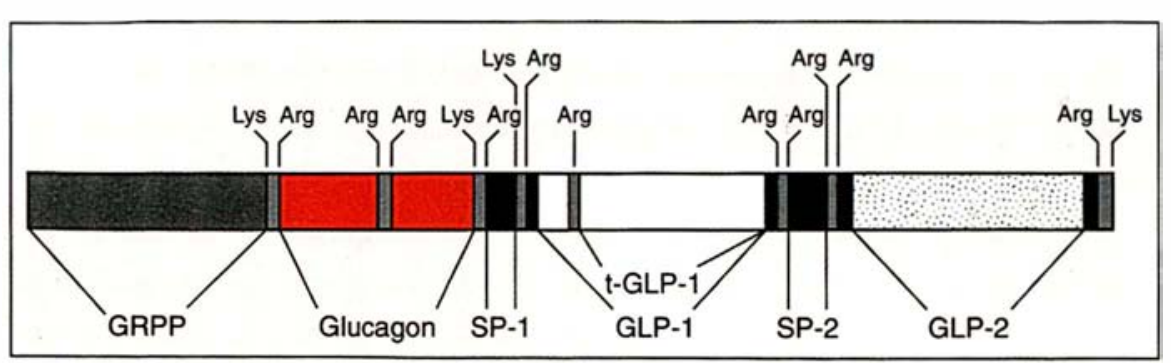

Figure 2. Structure du proglucagon humain. D'après Bell $[5,6]$. Les différents domaines (voir texte) sont séparés par des doublets basiques lysinearginine (Lys-Arg), arginine-arginine (Arg-Arg) ou arginine-lysine (Arg-Lys). Excepté quelques variantes, cette structure est commune à tous les 


\section{RÉFÉRENCES}

1. Bromer WW, Sinn LG, Behren OK. The amino acid sequence of glucagon. $\mathrm{V}$. Location of amide groups, acid degradation studies and summary of sequential evidence. J Am Chem Soc 1957 ; 79 : 2807-10.

2. Bataille D, Tatemoto K, Gespach C, Jörnvall $H$, Rosselin $G$, Mutt V. Isolation of glucagon-37 (bioactive enteroglucagon/oxyntomodulin) from porcine jejunoileum. Characterization of the peptide. FEBS Lett 1982 ; 146 : 79-86.

3. Thim L, Moody AJ. The primary structure of porcine glicentin (proglucagon). Regul Pept 1981; 2: 139-50.

4. Thim L, Moody AJ. Purification and chemical characterization of a glicentinrelated pancreatic peptide (proglucagon fragment) from porcine pancreas. Biochim Biophys Acta 1982 ; 703 : 134-41

5. Bell GI, Sanchez-Pescador R, Laybourn PJ, Najarian R. Exon duplication and divergence in the human preproglucagon gene. Nature $1983 ; 304: 368-71$.

6. Bell GI. The glucagon superfamily : precursor structure and gene organization. Peptides $1986 ; 7$ (suppl. 7) : 27-36.

7. Lund PK, Goodman RH, Dee PC, Habener JF. Pancreatic preproglucagon cDNA contains two glucagon-related coding sequences arranged in tandem. Proc Natl Acad Sci USA 1982 ; 79 : 345-9.

8. Blache P, Kervran A, Martinez J, Bataille D. Development of an oxyntomodulin/glicentin C-terminal radioimmunoassay using a " thiol-maleoyl " coupling method for preparing the immunogen. Anal Biochem 1988; 173 : 171-9.

9. Blache P, Kervran A, Bataille D. Oxyntomodulin and glicentin : brain-gut peptides in the rat. Endocrinology $1988 ; 132$ 2782-7.

10. Ravazzola M, Siperstein S, Moody AJ, Sundby F, Jacobsen H, Orci L. Glicentin immunoreactive cells and their relationship to glucagon-producing cells. Endocrinology 1979 ; 105 : 499-508.

11. Yanaihara C, Matsumoto T, Kadowaki M, Iguchi K, Yanaihara N. Rat pancreas contains the proglucagon (64-69) fragment and arginine stimulates its release. FEBS Lett 1985; 187 : 307-10.

12. Kervran A, Gibaud R, Pinet V Bataille D. Étude in vitro de la libération d'oxyntomoduline par le jéjuno-ileum de rat perfusé. Gastroenterol Clin Biol 1987; 11 vaux antérieurs [3, 10]. La maturation se poursuit par le clivage d'un hexapeptide à l'extrémité carboxyterminale de l'oxyntomoduline [11], peptide peu retenu par les colonnes et reconnu par l'anticorps noté "A " (figure 4).

Dans l'intestin (en fait dans les cellules $\mathrm{L}$ de la muqueuse), le type de maturation est très différent (figure 5). En effet, le glucagon est virtuellement

absent. Au contraire, l'oxyntomoduline, présente dans le pancréas à des taux de 5-15\% du contenu total en peptides de la famille, est ici le peptide dominant. Il est suivi de près par la glicentine. Cette proportion oxyntomoduline/glicentine est variable suivant les espèces (chez l'homme, les proportions sont inversées au profit de la glicentine) ; néanmoins, l'intestin contient toujours un

\section{RSLQDTEEKSRSFSASQADPLSDPDQMNEDKRHSQGTFTSDYSKYLDSRRAQDFVQWLMNTKRNRNNIA}

1

33

$6164 \quad 69$
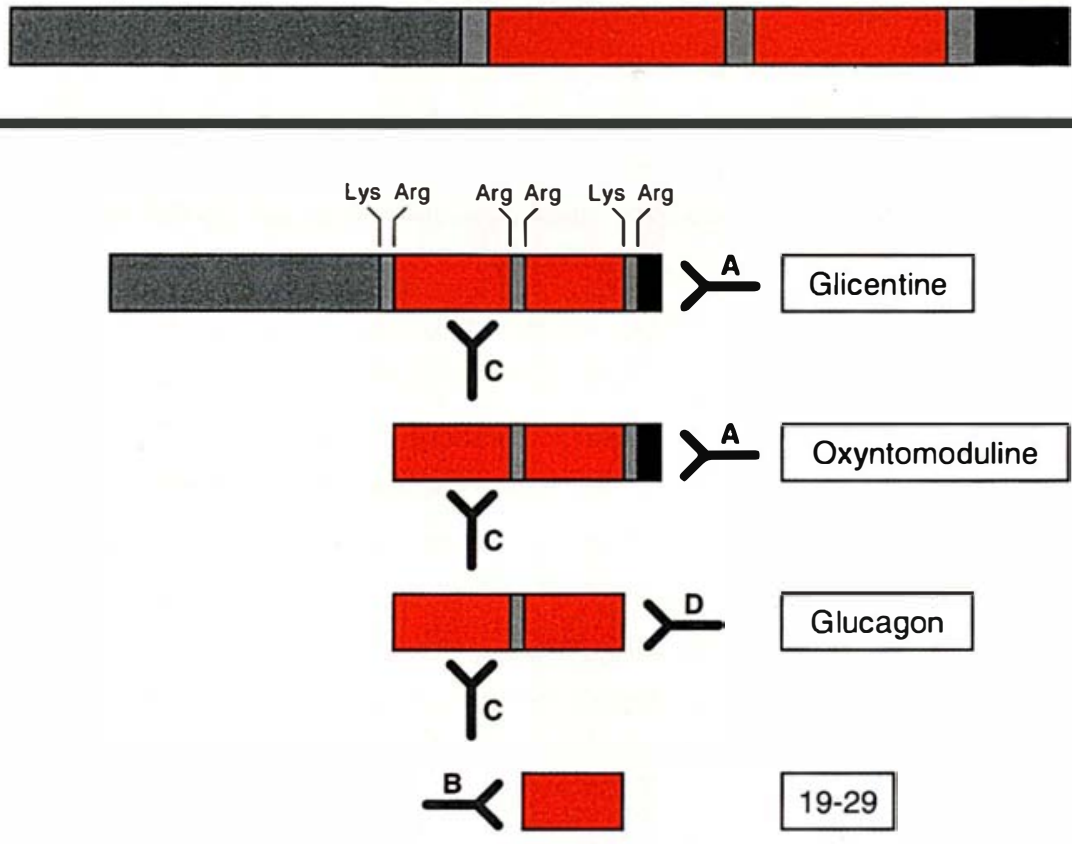

19-29

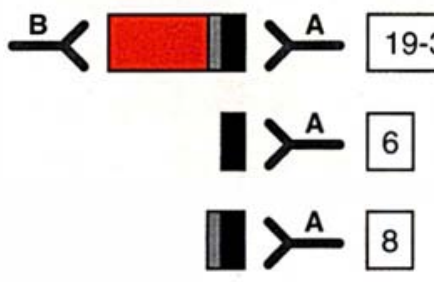

Figure 3. Les différents fragments potentiellement produits par maturation aux différents doublets basiques des 69 premiers acides aminés du proglucagon et qui ont été montrés comme d'importance biologique. Les six premiers sont produits soit dans les tissus producteurs : glicentine, oxyntomoduline, glucagon, hexapeptide (6), soit dans les tissus producteurs : 19-29, 19-37. L'octapeptide C-terminal (8) qui différencie l'oxyntomoduline du glucagon n'existe pas à l'état naturel mais présente un intérêt biologique considérable (voir texte). Les différents types d'anticorps produits contre des épitopes présents dans le précurseur $(C)$ ou démasqués lors des processus de maturation $(A, B, D)$ sont indiqués. Les anticorps $A$ et $B$ ont été obtenus grâce à des méthodes originales [8]. En haut : structure schématique et séquence en acides aminés (en code à une lettre) des fragments $\mathrm{N}$-terminaux du proglucagon : glicentine (1-69), oxyntomoduline (33-69), glucagon (33-61), hexapeptide (64-69). Les doublets basiques Lys-Arg (KR) ou Arg-Arg (RR) sont soulignés. 


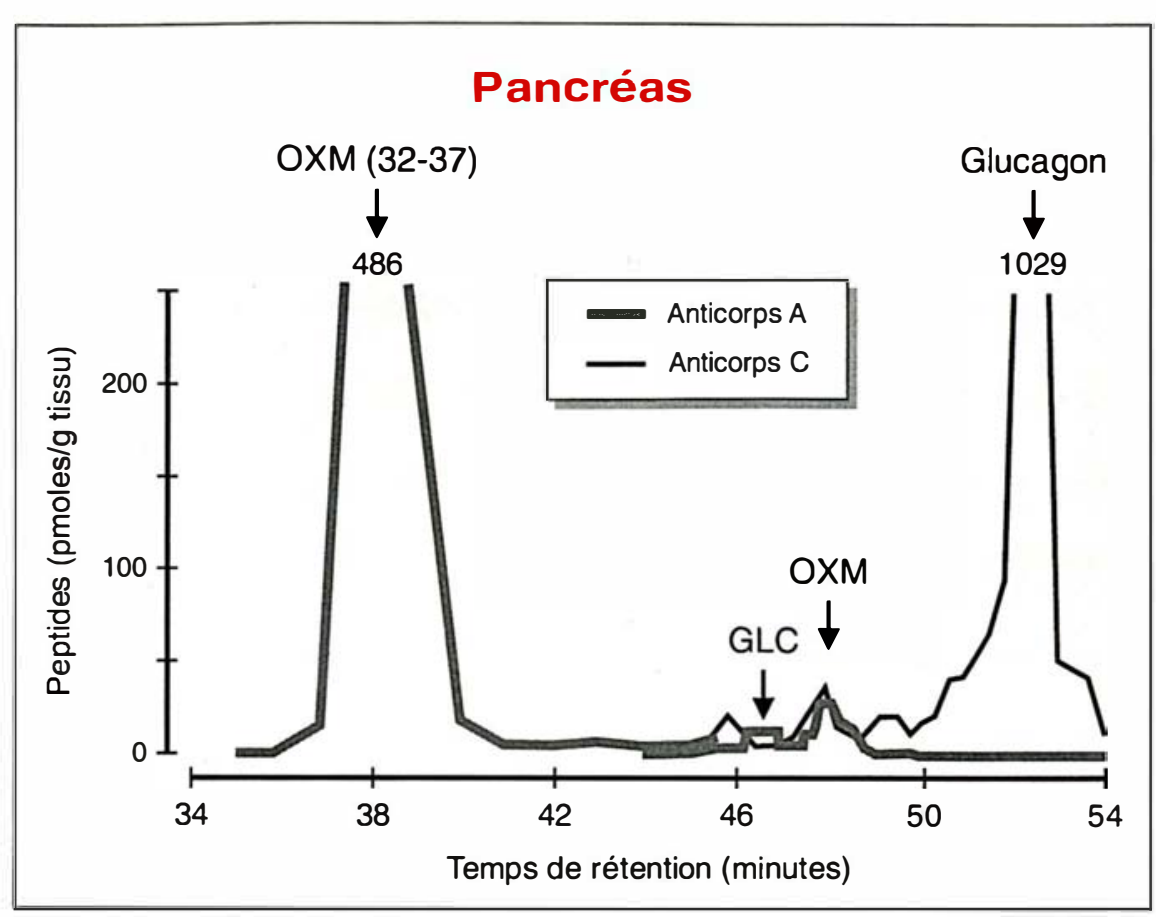

Figure 4. Analyse, par les anticorps $A$ et $C$ (voir figure 3 ) d'un extrait pancréatique de rat adulte chromatographié sur colonne de CLHP. L'OXM (32-37) correspond à l'hexapeptide, produit secondaire de la maturation de I'oxyntomoduline en glucagon (voir figures 3 et 6). Données tirées de [13].

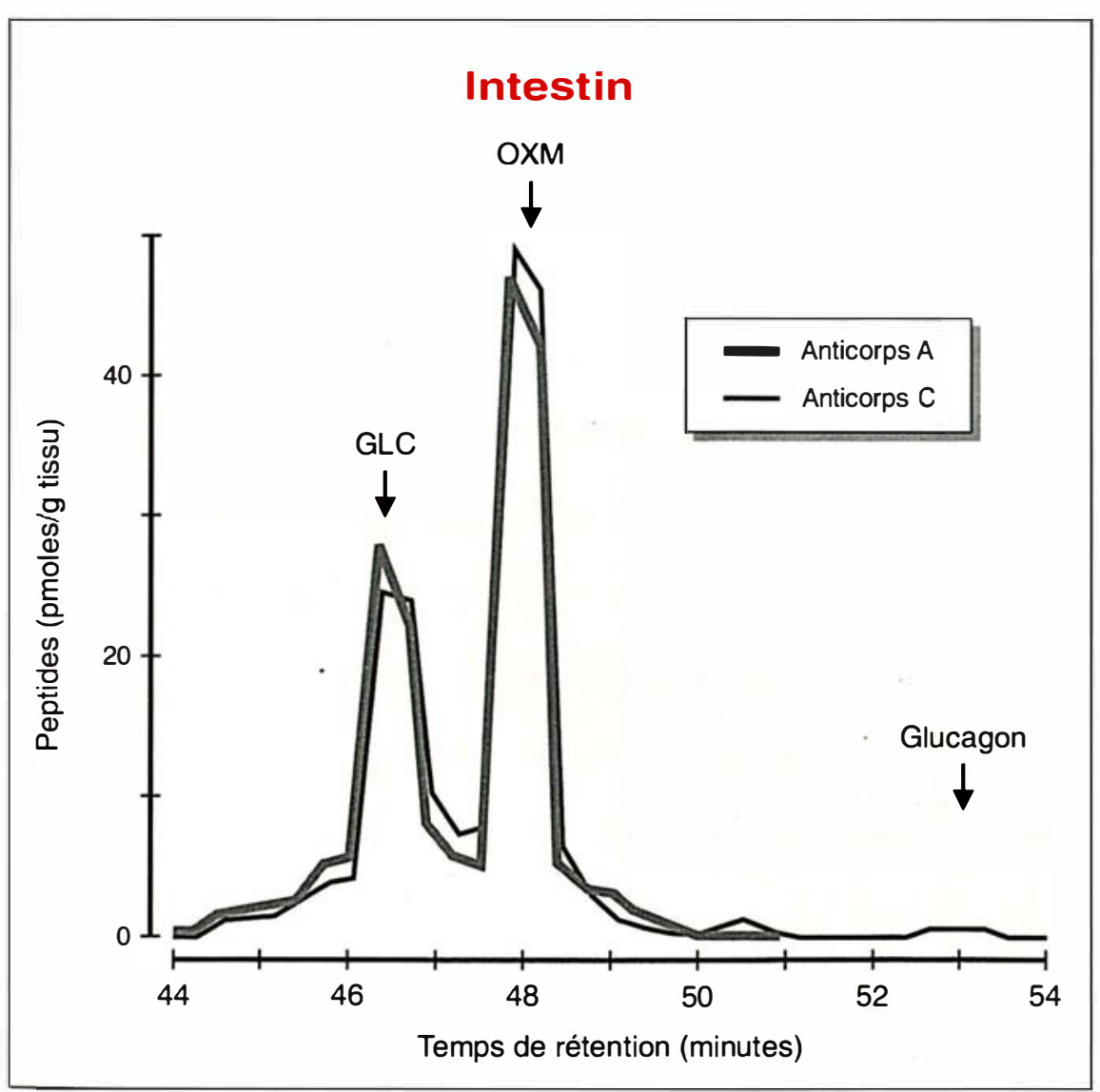

$\mathrm{m} / \mathrm{s} n^{\circ} \mathrm{9}$, vol. 7 , novembre 91 mélange des deux peptides. Il convient de noter que : i) les deux peptides sont stockés dans les grains de sécrétion et donc co-sécrétés, comme cela a été montré lors d'études menées sur un modèle d'intestin perfusé de rat [12]; ii) les deux peptides se trouvent dans la circulation systémique chez le rat à des taux supérieurs à ceux du glucagon et en proportion de leur concentration intestinale [13] ; iii) les deux peptides voient leur concentration doubler après une période de jeûne suivie d'une ré-alimentation, les taux de glucagon n'étant pas modifiés dans ces conditions [13]. Cet ensemble de données indique que l'ancien concept d'" entéroglucagon " ou "gut glucagon " [14] correspond, pour sa plus grande part, à un mélange d'oxyntomoduline et de glicentine. Cependant, ce concept immunologique [14] définit les molécules mises en évidence par les anticorps de type $\mathrm{C}$ (figure 3), qui reconnaissent toutes les molécules de la famille, y compris les formes prépro- et pro-, et non mises en évidence par les anticorps de type D (figure 3). En ce sens, il correspond non seulement aux molécules dans lesquelles l'épitope " $\mathrm{D}$ " est masqué par l'octapeptide (glicentine, oxyntomoduline et autres peptides plus longs) mais également dans lesquelles ce même épitope est absent, comme dans certaines formes plus courtes, tel le glucagon (1-21), qui présente des propriétés originales [15]. Comme indiqué plus loin, les formes contenant l'octapeptide Cterminal (oxyntomoduline et glicentine) se différencient nettement, sur le plan biologique, des formes ne le contenant pas (glucagon). Pour toutes ces raisons, il convient de remplacer le concept d'entéroglucagon par celui de peptides portant l'octapeptide.

Le type de maturation intestinale est présent également dans certaines zones du système nerveux central, en particulier l'hypothalamus et la

Figure 5. Analyse, par les anticorps $A$ et $C$ (voir figure 3 ), d'un extrait intestinal de rat adulte chromatographié sur colonne de CLHP. Conditions comme pour la figure 4 . 


\section{RÉFÉRENCES}

13. Kervran A, Blache P, Bataille D. Distribution of oxyntomodulin and glucagon in the gastro-intestinal tract and the plasma of the rat. Endocrinology $1987 ; 121$ : 704-13.

14. Unger RH. Circulating pancreatic glucagon and extrapancreatic glucagon-like materials. In : Handbook of Physiology. Endocrinology I. Amer Physiol Soc, 1972 : 529-44.

15. Matsuyama $\mathrm{T}$, Itoh $\mathrm{H}$, Watanabe $\mathrm{N}$, et al. Glucagon-(1-21)-peptide as an active enteroglucagon. Biomed Res 1988; 9 (Suppl 3) : 137-42.

16. Drucker DJ, Asa S. Glucagon gene expression in vertebrate brain. $J$ Biol Chem 1988 ; 263 : 13475-8.

17. Hoosein NM, Gurg RS. Identification of glucagon receptors in rat brain. Proc Natl Acad Sci USA 1984; 81 : 4368-72.

18. Bataille D. Gut glucagon. In : Schulz SG, Makhlouf G, Rauner BB. Handbook of Physiology - Section 6: The Gastrointestinal System Volume II. Neural and Endocrine Biology. Neuroendocrinology of the gut. Amer Physiol Soc, 1989 : 455-74.

19. Kervran A, Dubrasquet M, Blache P, Martinez J, Bataille D. Metabolic clearance rates of oxyntomodulin and glucagon in the rat : contribution of the kidney. Regul Peptides 1990 ; 31 : 41-52.

20. Bataille D, Gespach C, Coudray AM, Rosselin G. "Enteroglucagon " : a specific effect on gastric glands isolated from the rat fundus. Evidence for an "oxyntomodulin" action. Biosci Rep $1981 ; 1$ : 151-5.

21. Dubrasquet M, Bataille D, Gespach C. Oxyntomodulin (glucagon-37 or bioactive enteroglucagon): a potent inhibitor of pentagastrin-stimulated acid secretion in rat. Biosci Rep 1982 ; 2 : 391-5.

22. Jarrousse C, Audousset-Puech M-P, Dubrasquet M, Niel H, Martinez J, Bataille D. Oxyntomodulin (glucagon-37) and its C-terminal octapeptide inhibit gastric secretion. FEBS Lett $1985 ; 188 \div 81-4$.

23. Jarrousse C, Niel H, AudoussetPuech M-P, Martinez J, Bataille D. Oxyntomodulin and its C-terminal octapeptide inhibit liquid meal-stimulated acid secretion. Peptides 1986 ; 7 (Suppl. 1) : 253-6.

24. Kirkegaard P, Moody AJ, Holst JJ, Loud FB, Olsen PS, Christiansen J. Glicentin inhibits gastric acid secretion in the rat. medulla oblongata (située dans le bulbe rachidien). Deux éléments méritent d'être notés à ce niveau : i) l'ARN messager du proglucagon a été mis en évidence dans ces deux structures [16], cependant avec une nette prédominance pour la medulla oblongata. A l'inverse, pour ce qui concerne le contenu en peptides, l'hypothalamus est environ 250 fois plus riche que la medulla oblongata [9]. ii) A la différence de ce qui est observé dans l'intestin, une proportion non négligeable $(10-20 \%$ de l'ensemble) de glucagon est détectée [9]. Il est possible que certains neurones expriment une maturation de type intestinal, d'autres exprimant une maturation de type pancréatique; l'image finale viendrait de la juxtaposition de ces deux types de neurones dans les structures étudiées. Ces observations sont à mettre en parallèle avec la mise en évidence de récepteurs au glucagon dans le système nerveux central [17]. La figure 6 récapitule les données sur la maturation post-traductionnelle observée dans les tissus producteurs.

\section{Spécificité biologique des peptides portant l'octapeptide comparée au glucagon}

La comparaison directe des deux peptides dont la différence moléculaire est l'octapeptide (glucagon d'un côté, oxyntomoduline de l'autre) sur les mêmes modèles biologiques ont montré deux phénomènes intéressants : i) l'octapeptide gêne l'interaction de la molécule avec les récepteurs du glucagon mis en évidence dans de nombreux tissus (foie, tissu adipeux, cœur, rein...), puisque l'oxyntomoduline est active à des doses environ dix fois plus élevées que le glucagon sur ces tissus (figure 7) [2, 18]. Cela est vrai pour

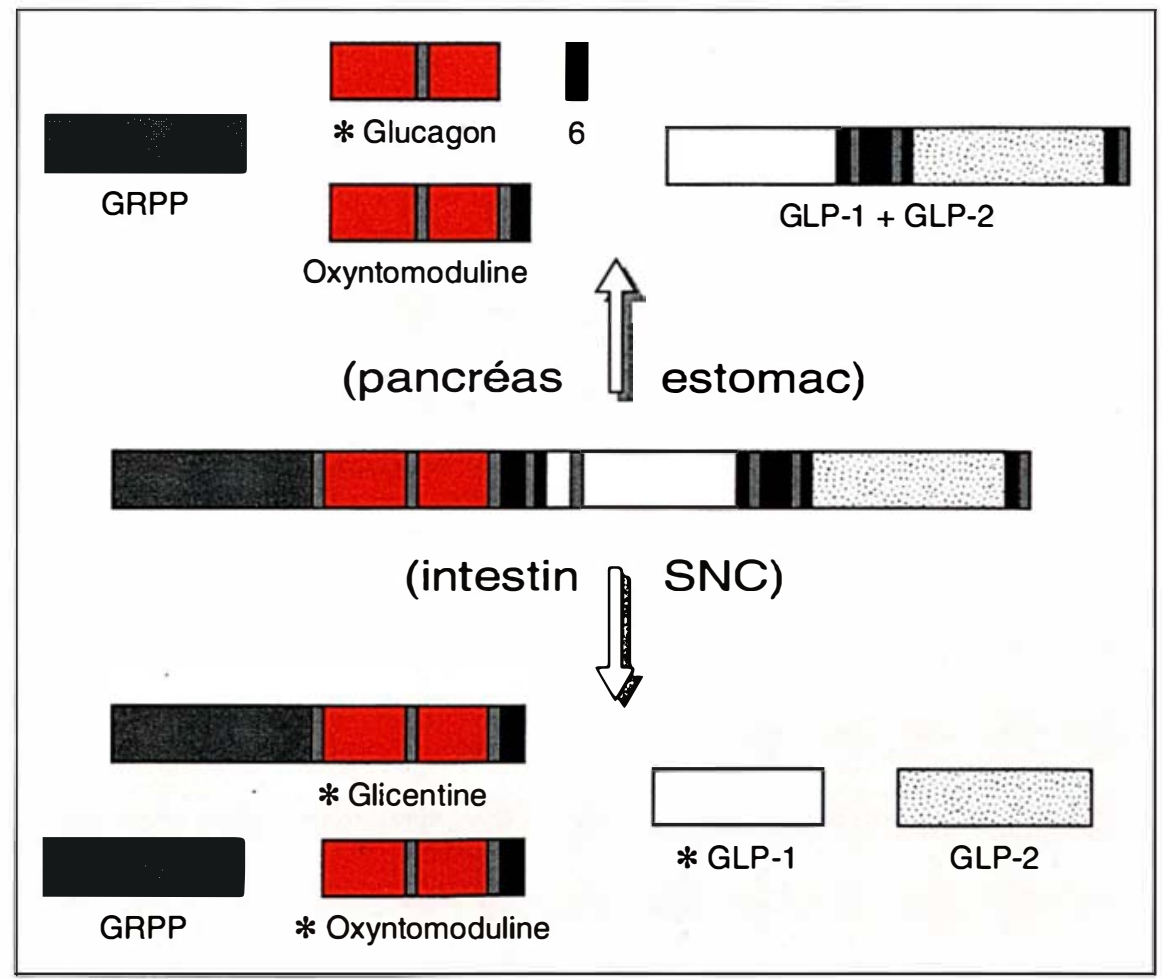

Figure 6. Récapitulation du type de maturation du proglucagon dans les tissus producteurs chez le rat : pancréas endocrine, muqueuse gastrique, muqueuse intestinale et système nerveux central (hypothalamus et medulla oblongata). Les produits de maturation les plus significatifs biologiquement sont marqués d'un astérisque. D'après les données publiées [9, 13]. 
l'interaction avec les récepteurs [2, 18], la stimulation de l'adénylate cyclase $[2,18]$ ou l'effet hyperglycémiant in vivo [19], compte non tenu des différences existant entre les demi-vies des peptides. Cela indique clairement que l'oxyntomoduline ne peut exercer d'action régulatrice physiologique via ces récepteurs. Un rôle exclusif de précurseurs biosynthétiques semblait être dévolu aux peptides portant l'octapeptide jusqu'à l'observation [20] qu'il existe un tissu dans lequel l'oxyntomoduline est le peptide le plus puissant : l'estomac. En effet, l'oxyntomoduline agit à des doses 10-20 fois plus faibles que le glucagon sur des glandes oxyntiques (contenant les cellules pariétales qui sécrètent l'acide, $0 \xi u v \tau o \sigma=$ acide) isolées de la muqueuse du fundus gastrique de rat (figure 7) [20]. Cette spécificité tissulaire originale suggère que ce peptide est un régulateur biologique au même titre que le glucagon, mais ayant un rôle certainement très différent. Cette spécificité tissu-

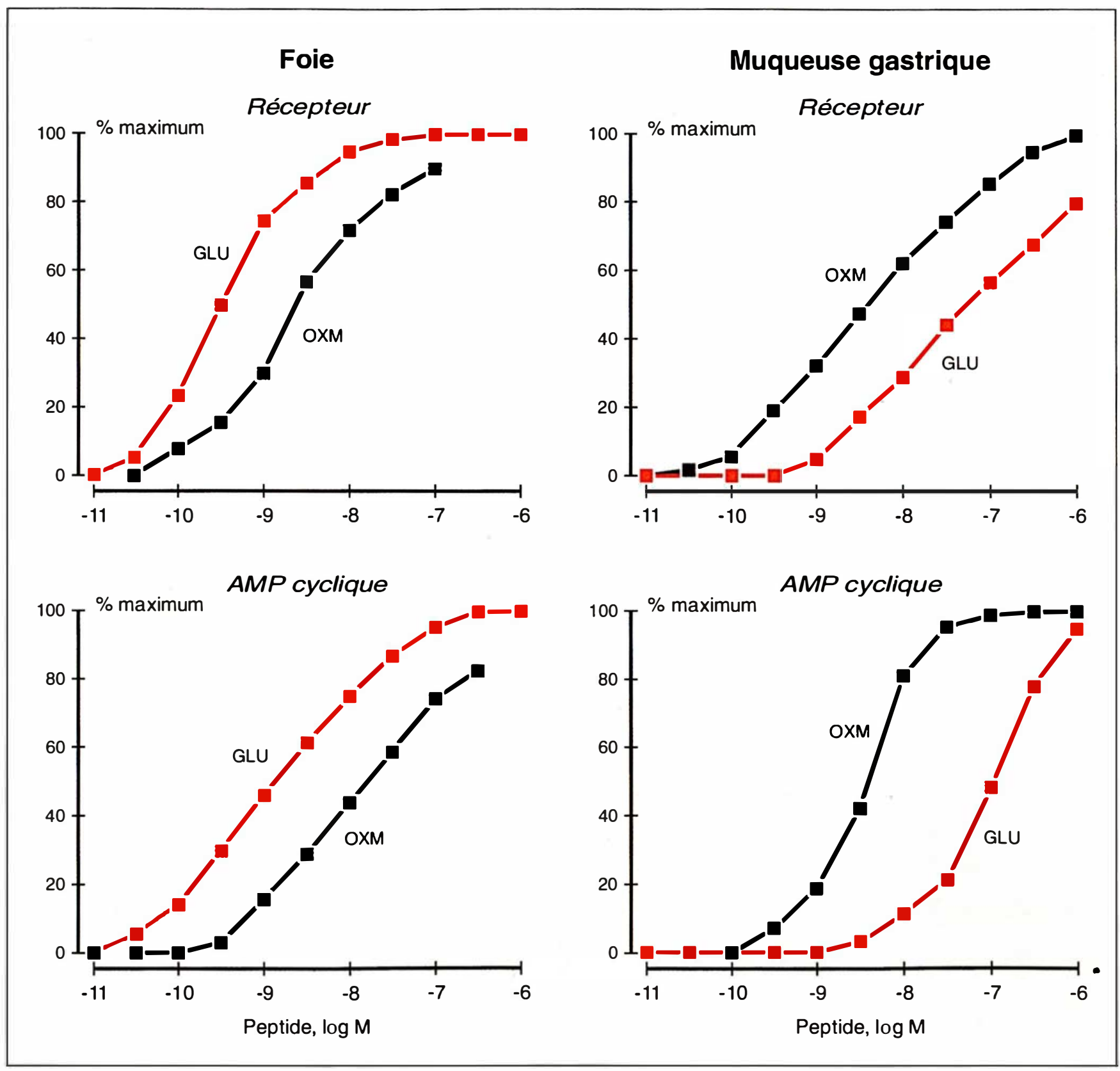

Figure 7. Comparaison des peptides ne contenant pas (glucagon, GLU) et contenant (oxyntomoduline, OXM) I'octapeptide C-terminal sur la liaison du peptide marqué (récepteur) et la stimulation d'AMP cyclique cellulaire (AMP cyclique) dans les membranes de foie de rat (panneaux de gauche) et dans les glandes oxyntiques isolées de la muqueuse du fundus gastrique de rat (panneaux de droite). Données tirées de [18, 20, 32]. 


\section{RÉFÉRENCES}

25. Schjoldager B, Baldissera FGA, Mortensen PE, Christiansen J, Holst JJ. Oxyntomodulin, a potential hormone from the distal gut. Pharmacokinetics and effects on gastric acid secretion in man. Eur J Clin Invest $1988 ; 18: 499-503$.

26. Veyrac M, Ribard D, Daures JP, et al. Inhibitory effect of the C-terminal octapeptide of oxyntomodulin on pentagastrinstimulated gastric acid secretion in man. Scand J Gastroenterol 1989 ; 24 : 1238-42.

27. Orskov C, Jeppesen J, Madsbad S, Holst JJ. Proglucagon products in plasma of noninsulin-dependent diabetics and non diabetic controls in the fasting state and after oral glucose and intravenous arginine. $J$ Clin Invest 1991; $87: 415-23$.

28. Robein MJ, De la Mare MC, Dubrasquet JM, Bonfils S. Utilization of the perfused stomach in anesthetized rats to study the inhibitory effect of somatostatin in gastric acid secretion. Agents Action $1979 ; 9$ : 415-21.

29. Dubrasquet J, Audousset-Puech MP, Martinez J, Bataille D. Somatostatin enhances the inhibitory effect of oxyntomodulin and its C-terminal octapeptide on acid secretion. Peptides 1986 ; 7 (Suppl 1) : 257-9.

30. Bado A, Bataille D, Accary JP, Lewin MJM, Dubrasquet M. Luminal gastric somatostatin-like immunoreactivity in response to oxyntomodulin and derivatives in cat. Biomed Res 1988; 9 (Suppl 3) : 195-9.

31. Tani T, Le Quellec A, Jarrousse C, et al. Oxyntomodulin and related peptides control somatostatin secretion in RIN T3 cells. Biochim Biophys Acta 1991; (sous presse).

32. Depigny C, Lupo B, Kervran A, Bataille D. Mise en évidence d'un site récepteur spécifique du glucagon-37 (oxyntomoduline/entéroglucagon bioactif) dans les glandes oxyntiques de rat. $C R$ Acad Sci Paris $1984 ; 299$ : 677-80.

33. Blache P, Kervran A, Dufour M, et al. Le glucagon est maturé en fragment (19-29) au niveau de la membrane hépatocytaire. CR Acad Sci Paris 1989; 17 : 467-72.

34. Blache $P$, Kervran A, Dufour M, et al. Glucagon (19-29), a $\mathrm{Ca}^{2}+$ pump inhibitory peptide, is processed from glucagon in the rat liver plasma membrane by a thiol endopeptidase. J Biol Chem 1990 ; 265 : 21514-9.

35. Rodbell M. The actions of glucagon at its receptor. In : Lefebvre PJ, ed. Glucagon. laire marquée pour la muqueuse oxyntique nous a amené à donner le nom d' " oxyntomoduline " au peptide intestinal [20]. Son activité biologique dirigée vers les mécanismes réglant la sécrétion acide est confirmée par les données obtenues chez le rat anesthésié [21] où l'oxyntomoduline est 15 fois plus puissante que le glucagon comme inhibiteur de la sécrétion acide stimulée. Des données obtenues sur des modèles plus physiologiques [22, 23], en particulier le rat éveillé dont la sécrétion gastrique est stimulée par un repas [23], ont renforcé le statut de l'oxyntomoduline comme régulateur physiologique négatif des phénomènes sécrétoires gastriques. Bien que les connaissances sur la glicentine soient beaucoup plus éparses, la molécule de 69 AA s'est avérée être également active sur la sécrétion acide [24]. Sur le plan pharmacologique, la structure la plus simple présente dans ces peptides qui soit capable de mimer l'activité inhibitrice est l'octapeptide Cterminal [22, 23], ce qui renforce encore le concept que les peptides portant l'octapeptide correspondent à une entité physiologique. L'ensemble des données en notre possession, en particulier les doses actives in vivo [22, 23] comparées aux taux circulants $[13,19]$, l'effet du peptide [25] ou de ses dérivés [26] chez l'homme ainsi que l'étude des rythmes nychtéméraux de ces peptides dans la circulation (A. Le Quellec, communication personnelle) permettent de conclure que l'oxyntomoduline (ou plus probablement l'ensemble oxyntomoduline + glicentine, c'est-à-dire les peptides sécrétés par l'intestin et portant l'octapeptide) sont impliqués dans les processus de rétro-contrôle des sécrétions gastriques. Il reste à déterminer dans quelle mesure des faiblesses de ces rétro-contrôles sont impliqués dans les hypersécrétions acides, aboutissant généralement aux ulcères duodénaux, et donc l'importance de l'oxyntomoduline et des peptides apparentés dans ces affections. L'équilibre entre les différents peptides dérivés du pro-

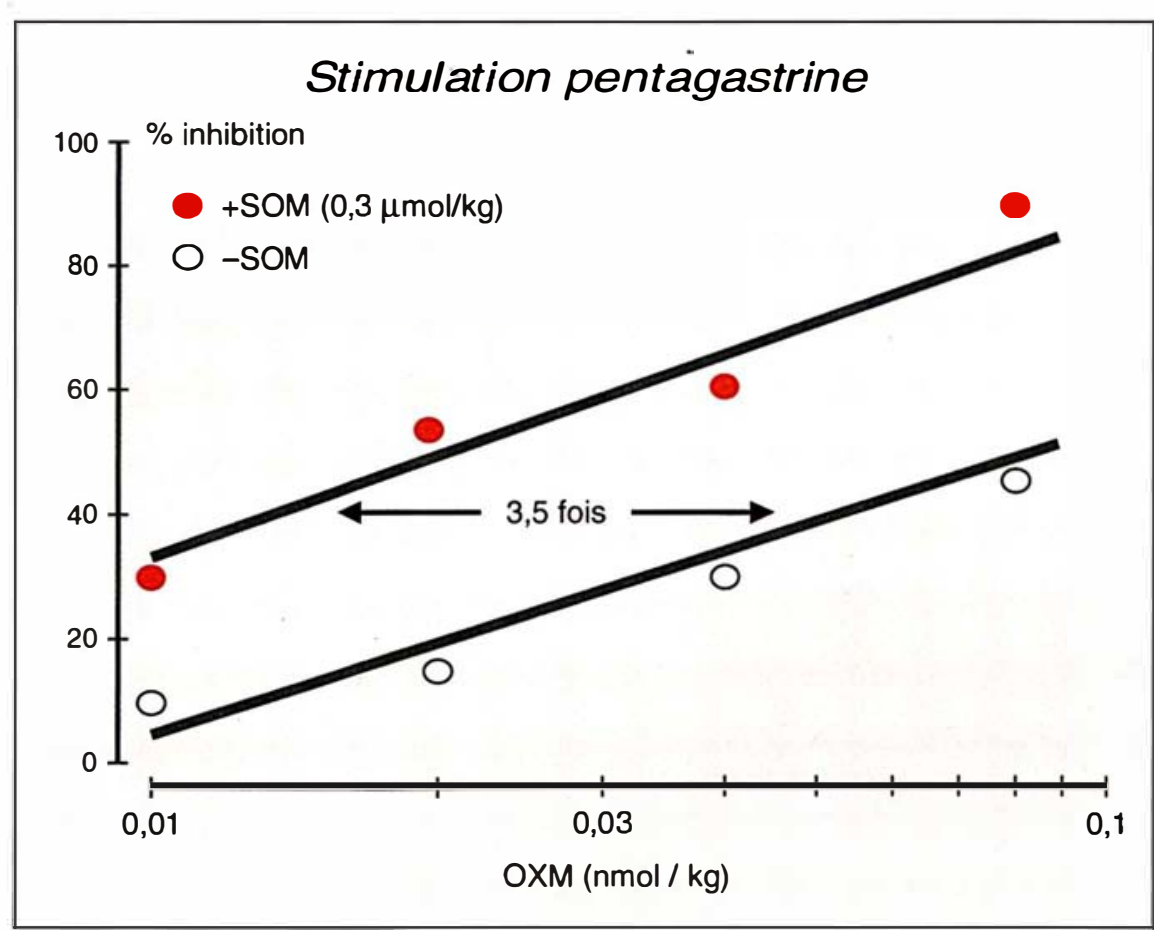

Figure 8. Potentialisation de I'effet inhibiteur de l'oxyntomoduline (OXM) sur la sécrétion acide gastrique induite par la pentagastrine chez le rat anesthésié par la somatostatine (SOM) utilisée à une dose sans effet par elle-même. Données tirées de [29]. 
glucagon peut être modifié dans certaines conditions chez l'homme, comme cela a été démontré chez les diabétiques insulinodépendants, sans que la signification de ces changements soit clairement comprise [27].

\section{Implication de mécanismes sécrétoires secondaires dans le mode d'action de ces peptides}

Le mode d'action, au niveau intégré, de l'oxyntomoduline implique une étape mettant en jeu la somatostatine, qui est connue comme inhibiteur de la sécrétion acide gastrique [28]. En effet, l'effet inhibiteur de l'oxyntomoduline sur la sécrétion acide induite par la pentagastrine chez le rat anesthésié est augmenté lorsque des doses, inefficaces par elles-mêmes, de somatostatine sont injectées [29]. Une potentialisation d'un facteur 3 à 4 de l'effet de l'oxyntomoduline est observée (figure 8). Lorsque l'histamine est utilisée comme stimulant, des doses croissantes de somatostatine, inefficaces par elles-mêmes, augmentent là encore l'effet de petites doses d'oxyntomoduline [29]. Par ailleurs, l'oxyntomoduline injectée induit une libération locale de somatostatine par les cellules $\mathrm{D}$ de la muqueuse gastrique [30]. Cet ensemble de données nous permet de conclure que l'oxyntomoduline déclenche, par l'intermédiaire de la somatostatine, un véritable mécanisme d'auto-potentialisation. La figure 9 schématise ce mécanisme, sur lequel nous ne possédons actuellement qu'une connaissance très partielle des systèmes cellulaires mis en jeu. Des données récentes [31], obtenues sur des cellules en culture sécrétant de la somatostatine, indiquent que des récepteurs à l'oxyntomoduline couplés à l'adénylate cyclase et aboutissant à la libération de somatostatine existent dans les cellules $\mathrm{D}$ gastriques (figure 9). Ces récepteurs sont sans doute ceux qui ont été découverts dans les glandes gastriques isolées $[18,32]$ et qui ont été à l'origine du concept même d'oxyntomoduline, grâce au système de second messager couplé à ces récepteurs [20]. Cela n'exclut pas que ces récepteurs puissent exister également dans d'autres $m / s n^{\circ} 9$, vol. 7 , novembre 91

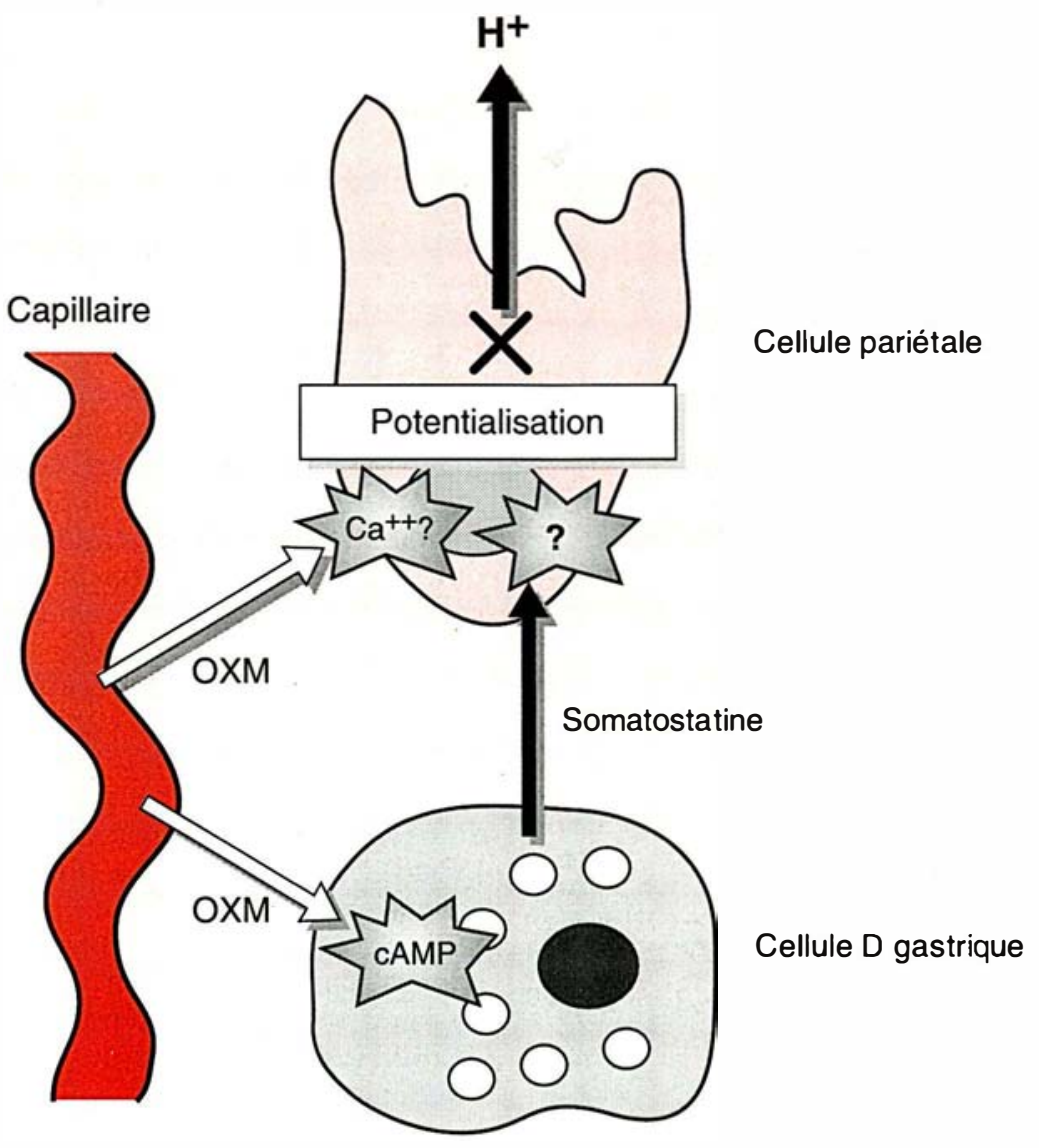

Muqueuse gastrique

Figure 9. Schéma des événements se produisant dans la muqueuse oxyntique de rat lors de l'arrivée par voie systémique d'oxyntomoduline (OXM). La cellule $D$ gastrique est la cellule à somatostatine de la muqueuse, proche de la cellule pariétale qui sécrète l'acide grâce à la pompe à protons $\left(H^{+}, K^{+}\right.$ATPase $)$.

types cellulaires de la muqueuse fundique. Les mécanismes de potentialisation existant au niveau de la cellule pariétale (figure 9) font actuellement l'objet d'études. Il est possible que les variations de $\mathrm{Ca} 2+$ cytosolique soient impliquées dans ces mécanismes.

\section{Mutation des peptides circulants au niveau des tissus cibles}

Compte tenu des types de fragments de proglucagon présents dans les tissus producteurs (figure 3), tous les doublets basiques présents dans le précurseur apparaissent, dans certaines circonstances, coupés par des enzymes de maturation au sein des tissus producteurs, exception faite du doublet Arg-Arg présent en position 17-18 du glucagon (ou de l'oxyntomoduline). Cependant, il est clair, désormais, que ce site potentiel de maturation est effectivement la cible d'enzymes présentes dans les membranes de certains tissus cibles et que le substrat de ces enzymes est le peptide circulant ; la différence apparaît donc clairement avec ce qui se passe dans les tissus producteurs, où le substrat des enzymes de maturation est le proglucagon.

\section{- Glucagon}

Nous savons maintenant que la maturation du glucagon peut se 


\section{RÉFÉRENCES}

36. Sheetz MJ, Tager H. Characterization of a glucagon receptor-linked protease from canine hepatic plasma membranes. $J$ Biol Chem 1988 ; 263 : 19210-7.

37. Rose K, Savoy LA, Muir AV, Davies JG, Offord RE, Turcatti G. Insulin proteinase liberates from glucagon a fragment known to have enhanced activity against $\mathrm{Ca}^{2+} \mathrm{Mg}^{2+}$-dependent ATPase. Biochem J 1988 ; 256 : 847-51.

38. Duckworth W. Insulin degradation : mechanisms, products, and significance. Endocrine Rev 1988; 9: 319-45.

39. Mallat A, Pavoine C, Dufour M, Lotersztajn S, Bataille D, Pecker F. A glucagon fragment is responsible for the inhibition of the liver $\mathrm{Ca}^{2}+$ pump by glucagon. Nature $1987 ; 325: 620-2$

40. Loterszta jn S, Pavoine C, Brechler V, et al. Glucagon (19-29) exerts a biphasic action on the liver plasma membrane $\mathrm{Ca}^{2}+$ pump which is mediated by $\mathrm{G}$ proteins. J Biol Chem 1990; 265 : 9876-80.

41. Lotersztajn S, Epand RM, Mallat A, Pecker F. Inhibition by glucagon of the calcium pump in liver plasma membranes. $J$ Biol Chem 1984; 259 : 8195-201.

42. Pavoine C, Brechler V, Kervran A, et al. Miniglucagon, glucagon-(19-29), is a component of the positive inotropic effect of glucagon. Am J Physiol 1991 ; 260 : C993-9.

43. Bataille D, Jarrousse C, Blache P, Kervran $\mathrm{A}$, et al. Oxyntomodulin and glucagon : are the whole molecules and their Cterminal fragments different biological entities? Biomed Res 1989; 9 (Suppl 3): 169-79.

44. Jarrousse C, Mercier F, Martinez J, Bataille D. Oxyntomodulin (glucagon-37) : effect of the 19-37 fragment on gastric acid secretion. 1st Eur Congress of Endocrinology, Copenhagen, 1989 ; 65 (Abstract).

45. Namba M, Itoh $\mathrm{H}$, Watanabe $\mathrm{N}$, et al. Multiple forms of glucagon-like peptide-1 and glucagon-like immunoreactivities in canine gastrointestinal tract and their release into circulation. Biomed Res $1990 ; 11$ : 247-54.

46. Mojsov S, Kopczynski MG, Habener JF. Both amidated and nonamidated forms of glucagon-like peptide I are synthesized in the rat intestine and the pancreas. J Biol Chem 1990 ; 265 : 8001-8.

47. Cohen P. Proteolytic events in the posttranslational processing of polypeptide hormone precursors. Biochimie 1987; 69 : 87-9. dérouler in vitro dans les membranes de foie de rat en fragment (19-29) [33, 34] ; elle est catalysée par une enzyme, liée à la membrane cellulaire, qui a les caractéristiques d'une thiol-endopeptidase comportant un ion métallique dans son site actif [34]. Cette enzyme n'est pas liée au récepteur du glucagon (le récepteur couplé à l'adénylate cyclase [35]). Les deux systèmes, reconnaissance du glucagon par ses récepteurs et production du fragment, sont indépendants*, ce qui crée certainement un avantage fonctionnel. Le fragment (18-29), apparemment produit à partir de glucagon par action d'une autre thiol-métallo-endopeptidase [37], l'insulin degrading enzyme [38], ne peut servir de substrat à l'enzyme qui produit le (19-29) [34]. Enfin, le fragment (19-29) ainsi produit est rapidement inactivé par une autre enzyme de la classe des aminopeptidases [34]. Entre sa production et son inactivation, qui sont très proches dans le temps et probablement dans l'espace, le fragment a le temps de jouer son rôle qui semble être la modulation de l'activité du glucagon lui-même via un réglage adéquat de la pompe à $\mathrm{Ca} 2+$ de la membrane plasmique. En effet, le fragment (19-29) s'est avéré être actif sur cette pompe (ATPase à $\mathrm{Ca} 2+$ dont le rôle est de maintenir le gradient de concentration en $\mathrm{Ca}^{2}+$ entre le milieu extracellulaire et le cytosol) à des doses sub-nanomolaires [39, 40], le glucagon étant actif à des doses micromolaires sur ce système [41] ${ }^{* *}$. Le lien entre la reconnaissance du fragment (19-29) par son récepteur supposé et la pompe est un système de protéine(s) $G$, la présence de GTP (ou d'analogues du nucléotide) modulant l'effet du fragment sur la pompe [40]. Le récepteur du (19-29), dont la présence sur les membranes plasmiques est suggérée par ces données, n'a pas encore été mis en évidence.

Il apparaît clairement que la production du fragment (19-29) est importante dans le mode d'action du glucagon lui-même, puisque, comme

\footnotetext{
* Une enzyme de dégradation, liée au récepteur du glucagon et produisant le fragment (14-29) a été mise en évidence dans les membranes de foie [36].

** Voir aussi sur ce sujet l'article de F. Pecker, p. 911 de ce numéro.
}

cela a été montré récemment [42], la présence du fragment potentialise l'effet inotrope positif du glucagon sur des myocytes de cœur de poulet en culture. Ce même modèle a permis de montrer que le glucagon est transformé en fragment (19-29) au niveau du cœur [42]. Des données non publiées indiquent qu'un effet potentialisateur de l'effet du glucagon par le fragment existe également dans l'hépatocyte, où la maturation a été démontrée [33, 34].

Cet ensemble de connaissances indique qu'intervient, dans le mode d'action du glucagon, la production dans les tissus cibles du fragment (19-29) par maturation de l'hormone par une peptidase spécifique (en cours d'isolement et de caractérisation dans notre laboratoire) ; les conséquences de cette maturation sont une modification des concentrations de l'ion $\mathrm{Ca}^{2}+$ libre via un récepteur spécifique (non encore caractérisé) qui permet une modulation de l'activité de la pompe à $\mathrm{Ca}^{2}+$ et, en conséquence, une potentialisation de l'effet de l'hormone-mère dont le second messager est l'AMP cyclique [35]. La figure 10 schématise ce modèle.

\section{- Oxyntomoduline}

Un modèle un peu différent, bien que proche, semble exister pour l'hormone de $37 \mathrm{AA}:$ la même enzyme hépatique qui assure la maturation du glucagon [34] semble être active sur l'oxyntomoduline [43]. Cependant les conséquences de cette maturation ne sont pas identiques à celles du modèle "glucagon ". En effet, le fragment produit, OXM (19-37), équivalent du glucagon (19-29), ne potentialise pas l'effet de l'OXM, mais semble plutôt représenter un second messager, lui-même hormonal : le fragment (19-37) semble sensiblement aussi actif que l'hormone d'origine sur la sécrétion acide $[43,44]$. Ce fragment présente également le même type d'action sur la sécrétion de somatostatine que l'OXM [30, 31]. Tout ce qui a été dit plus haut à propos de l'OXM sur les mécanismes sécrétoires peut donc s'appliquer au fragment (19-37) qui pourrait constituer le messager final intercellulaire des peptides portant l'octapeptide. Cela expliquerait que la proportion d'OXM par rapport à 


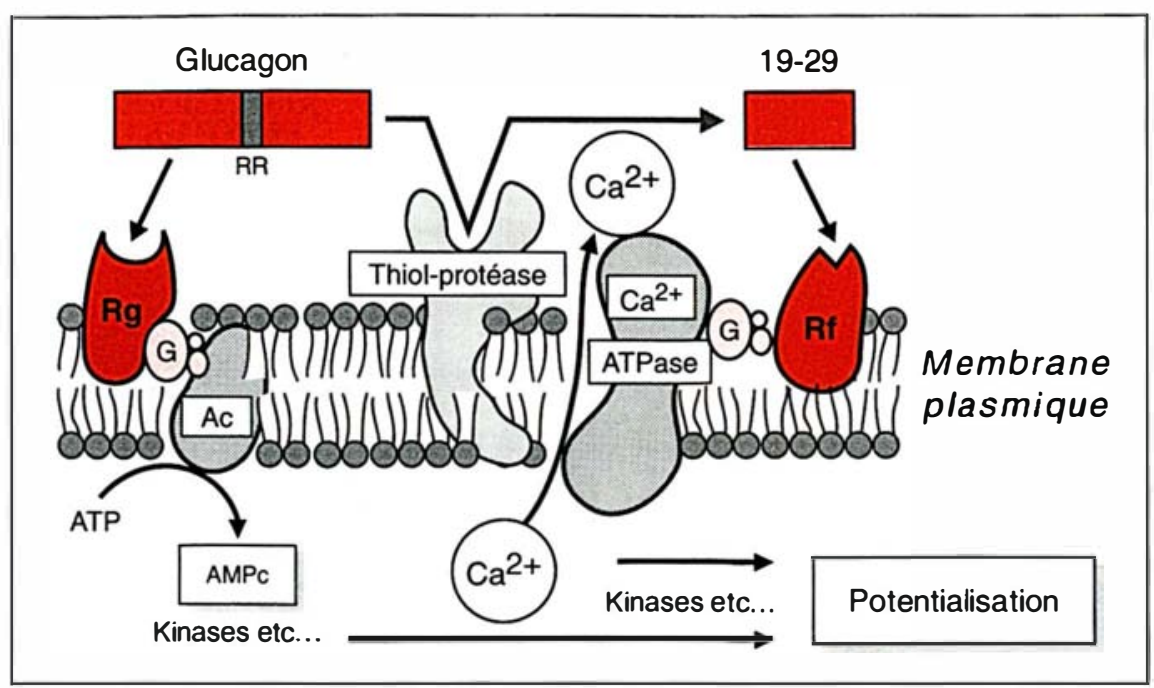

Figure 10. Schéma probable des événements se produisant au niveau de la membrane des cellules cibles du glucagon. Rg : récepteur du glucagon [35] couplé à l'adénylate cyclase (Ac) par une protéine liant le GTP (G). Le glucagon est clivé au niveau du doublet basique par une thiolprotéase [34], liée à la membrane mais indépendante du récepteur du glucagon, libérant le fragment C-terminal (19-29). Ce fragment, par l'intermédiaire d'un récepteur spécifique (Rf) non encore mis en évidence, lié à une protéine liant le GTP (G) [40], module l'activité de la pompe à $\mathrm{Ca}^{2+}\left(\mathrm{Ca}^{2+}\right.$ ATPase) [39]. Les variations de vitesse de pompage aboutissent dे des changements de concentration cytosolique de $\mathrm{Ca}^{2+}$ libre, et, en conséquence, de l'activité de kinases $\mathrm{Ca}^{2+}{ }^{+}$-dépendantes. L'activation des voies kinase $\mathrm{A}$ et kinase $C$ aboutit à l'effet potentialisateur observé [42].

la glicentine varie selon les espèces sans conséquence apparente, les deux molécules étant transformées en fragment (19-37), molécule active in fine. $\mathrm{Si}$ cette hypothèse s'avère exacte, le rôle du récepteur de l'OXM couplé à l'adénylate cyclase serait spécifique de la cellule D. Le fragment (19-37) n'a, pour sa part, aucun effet sur ce récepteur ni sur les taux d'AMP cyclique de la muqueuse gastrique ou des cellules D [31, 43, 44]. Comme son équivalent glucagon (19-29), l'OXM (19-37) semble posséder un récepteur propre qui pourrait être relié à un système de type $\mathrm{Ca}^{2}+$ ne mettant pas en jeu la phospholipase C [31].

\section{Conclusions}

Les peptides dérivés du proglucagon offrent un vaste éventail de mécanismes de régulation biologique. Au même titre que l'insuline, le glucagon a été, depuis sa découverte, un peptide modèle qui a servi à mettre sur pied un grand nombre de théo$\mathrm{m} / \mathrm{s} n^{\circ} 9$, vol. 7 , novembre 91 l'autre produisant un mélange de deux hormones co-sécrétées par l'intestin et contrôlant les sécrétions gastriques au niveau périphérique et des phénomènes encore à découvrir au niveau du système nerveux central. L'octapeptide porté en Cterminal par ces molécules leur confère des propriétés nouvelles tout en faisant disparaître en partie les propriétés de la molécule de glucagon. En ce sens, la maturation dans le tissu producteur du même précurseur (ce qui constitue une économie pour l'organisme) effectue un travail de sélection aux conséquences considérables puisqu'elle aboutit à des entités endocriniennes parfaitement différenciées.

Un point particulier concerne la découverte récente de la maturation post-traductionnelle au niveau de tissus cibles [33, 34, 42, 43], produisant, dans le cas du glucagon, un fragment C-terminal dont le rôle semble être d'amplifier la réponse du tissu cible à l'hormone mère. Dans le cas des peptides portant l'octapeptide, les hormones mères sont deux, et il semble que le peptide produit (peut-être en utilisant la même enzyme que dans le cas du glucagon) soit une nouvelle hormone fille qui effectue le travail final de déclenchement de l'activité biologique dans le tissu cible.

Il est intéressant de noter que, pour les deux types de peptides (glucagon d'une part et peptides portant l'octapeptide d'autre part), il existe un système amplificateur, originaire, du signal moléculaire initial, qui potentialise l'effet de ce dernier. Dans le premier cas, c'est un fragment de l'hormone elle-même qui est le signal secondaire ; dans le second cas, c'est un autre peptide (la somatostatine) dont la libération est contrôlée par l'hormone d'origine (comparer figures 9 et 10).

Il est probable que ces processus complexes, mais, somme toute, économiques pour la nature car ils réutilisent à d'autres fins des structures et des mécanismes déjà existants, répondent à un besoin, de plus en plus pressant au cours de l'évolution, en processus régulateurs de plus en plus rigoureux

TIRÉS A PART

D. Bataille

(Summary p. suivante) 909 


\section{Summary}

Proglucagon-derived peptides : post-translational processings and their biological consequences

Proglucagon, a 160 aminoacid (AA)-protein synthesized in endocrine pancreas, gastric mucosa, intestinal mucosa and the central nervous system (CNS), contains several domains separated by dibasic pairs. The first $69 \mathrm{AA}$, glicentin, includes oxyntomodulin (OXM, $37 \mathrm{AA}$ ) and glucagon (GLU, 29 AA) ; the difference between the two is a C-terminal basic octapeptide. Two other domains contain glucagon-like peptides, GLP-I and II which have their own biological pathways. Inside the glucagon-containing domain, two types of processing occur in the producing tissues, one leads to GLU (pancreas and stomach), the other leads to a mixture of the octapeptide-containing peptides, glicentin and OXM (intestine, CNS). The presence of the octapeptide decply modifies the biological specificity of the peptide : OXM is a hormone which regulates gastric acid secretion, the GLU specificity is directed towards the tissues that regulate metabolism and towards cardio-vascular and renal systems. For both types of peptides (octapeptide-containing and octapeptide-free), a potentiation system exists : the first type induces a local somatostatin release which potentiates the inhibitory effect of the hormone. For the second type, a processing by a thiol-endopeptidase at a dibasic site of the hormone occurring in the target tissue releases a C-terminal fragment (19-29) which modulates the cellular $\mathrm{Ca}^{2}+$ concentration via the plasma membrane $\mathrm{Ca}$ ?. ATPase, leading to a potentiation of the effect of the hormone which uses CAMP as the second messenger. In the first type of peptides, the same processing seems to occur, the C-terminal fragment (19-37) being the final hormonal messenger. 